\title{
tic\&société
}

Vol. 10, N 2-3 | 2ème semestre 2016 - 1er semestre 2017

Spécial varia

\section{Épuiser sa présence : rapports amoureux et téléphonie mobile}

Alexandre ROUXEL, Romain HUËT et Olivier SARROUY

\section{OpenEdition}

1 Journals

Édition électronique

URL : http://journals.openedition.org/ticetsociete/2184

DOI : 10.4000/ticetsociete. 2184

Éditeur

Association ARTIC

Édition imprimée

Pagination : 9-44

Référence électronique

Alexandre ROUXEL, Romain HUËT et Olivier SARROUY, «Épuiser sa présence : rapports amoureux et téléphonie mobile», tic\&société [En ligne], Vol. 10, № 2-3 | 2ème semestre 2016 - 1er semestre 2017, mis en ligne le 30 avril 2017, consulté le 19 avril 2019. URL : http://journals.openedition.org/ ticetsociete/2184; DOI : 10.4000/ticetsociete.2184 
tic\&société - 10(2-3), 2016-2017

\title{
Épuiser sa présence : rapports amoureux et téléphonie mobile
}

\author{
Alexandre ROUXEL, Romain HUËT et Olivier SARROUY
}

Alexandre Rouxel est doctorant en sciences de l'information et de la communication à l'université Rennes 2 . Sa thèse porte sur l'aide par messagerie instantanée dans le cadre des actions d'accompagnement de la souffrance à distance. Dans le cadre de cette thèse, ses travaux de recherche portent sur les formes contemporaines de la coprésence numérique. Ses différentes analyses visent ainsi à éclairer les effets du web et des technologies de communication numérique sur les formes du rapport à soi et sur les manières d'être ensemble à distance. rouxelalexandr@gmail.com

Romain Huët est maître de conférences en SIC à l'université Rennes 2. Il est chercheur au Prefics (université Rennes 2) et à l'Institut supérieur de la communication (CNRS/Paris Sorbonne). Son travail porte sur les liens entre souffrance sociale et révolte. II a notamment travaillé à une ethnographie des moudjahidines syriens et sur le suicide en France. Son hypothèse centrale consiste à penser que les luttes sociales prennent leur lit sur le constat du caractère injustifié, douloureux, voire intolérable, de l'existence. Ce travail s'appuie sur diverses recherches empiriques pour saisir comment des " espaces morales de l'intolérable » se constituent: énonciation de la souffrance ordinaire au sein de dispositifs technologiques de prévention du suicide et ethnographie des lieux de contestation armée (Syrie, Ukraine, Irak). rhhuet@gmail.com

Olivier Sarrouy est maître de conférences en SIC à l'université Rennes 2. Ses recherches portent sur les recompositions économiques, organisationnelles, sociales et politiques qu'exerce aujourd'hui l'hégémonie du web. Ses analyses visent à éclairer les manières dont le web affecte les formes de l'être et de l'agir-en-commun. Ce travail s'appuie sur diverses recherches empiriques: mutations du web, évolution des formes d'organisation pair-à-pair, mutation des relations sous l'effet des technologies numériques, expression en ligne du soi, etc. Ses réflexions s'orientent désormais vers la possibilité de penser une communauté politique invalidant l'hypothèse théorique et institutionnelle de l'unité du corps social ou politique. olivier.sarrouy@gmail.com 


\section{Épuiser sa présence : rapports amoureux et téléphonie mobile}

Résumé : Cet article examine les spécificités des rapports amoureux actuels en tant que ceux-ci sont équipés par toute une série de technologies à même d'organiser concrètement le contenu des relations. II en est ainsi du téléphone portable, qui s'est immiscé dans le cœur de la vie amoureuse. Aussi, au moyen d'une phénoménologie de la relation amoureuse, il s'agira d'examiner les recompositions des régimes présentiels et relationnels engendrés par le téléphone portable. À partir d'un corpus de 4974 messages instantanés échangés par neuf couples distincts, le but est d'appréhender la fragilisation du mouvement vers autrui que pourrait entraîner l'utilisation de ces technologies, dont l'effet essentiel est probablement d'organiser une coprésence particulière des êtres.

Mots-clés : relations amoureuses, téléphone portable, SMS, passion, coprésence.

Abstract: This article analyzes the recomposition of presential
and relational regimes caused by the mobile phone in love
relationships. We will discuss phenomenological specificities of
in-love copresence before examining results that introduce the
effects of "availability" raised by these devices. This analysis
will be based on a corpus of 4,974 SMS exchanged by nine
different couples. We will especially highlight the specificities of
the "technical principle" inherent to these devices. The analysis
of this "short time technology" helps to discern a radicalization
of the feeling of remotness to the other.in this "technologically
equipped copresence."

Keywords: love relationships, cellphone, SMS, passion, copresence.

Resumen: Este artículo pretende analizar las especificidades de las relaciones amorosas en la actualidad, en la medida en que estas utilizan una serie de tecnologías, que incluso llegan a organizar el contenido de las relaciones. El mejor ejemplo es el teléfono móvil, que se ha situado en el centro de la vida amorosa. Mediante una fenomenología de la relación amorosa, se busca analizar las recomposiciones de los regímenes presenciales y relacionales generados por el teléfono móvil. Este análisis se apoya en un corpus de 4974 mensajes de texto (SMS) intercambiados por 9 parejas distintas. De manera más específica se busca analizar la fragilización del movimiento 
Épuiser sa présence : rapports amoureux et téléphonie mobile

hacia la otra persona que pudiera resultar de la utilización de estas tecnologías con probabilidad, el efecto esencial es el de organizar una co-presencia particular de las personas.

Palabras-clave: relaciones amorosas, teléfono móvil, SMS, pasión, copresencia. 



\section{Rapports amoureux et technologies}

Dans un rapport amoureux, le téléphone portable peut parfois susciter d'étranges interactions: messages insistants, réponses vexées, volonté de contrôle total sur l'autre, sa situation, ou encore ses rencontres, etc. Ceux qui ont vécu une relation (un peu trop) passionnelle pourront ainsi reconnaître l'étrangeté de ces messages qu'ils se seront surpris avoir envoyés: "pourquoi ne me réponds-tu pas ? ", " et voilà, tu dois être occupé(e) à mieux que moi », " tu t'en fiches que je t'écrive ou quoi ? ", "ça fait une heure que j'essaye de te joindre ! ", "Tu es avec quelqu'un d'autre, c'est ça? ", etc. Peut-être la tonalité de ces messages s'explique-t-elle par la nature si particulière des rapports amoureux contemporains. Notre hypothèse est que cette explication est insuffisante. II existe également un principe technique à l'organisation de la présence entre les partenaires qui pourrait susciter une certaine façon de se rendre mutuellement présent l'un à l'autre. II importe alors de saisir la manière dont le téléphone organise le rapport social entre les partenaires amoureux. Plus précisément, il sera montré que le téléphone portable induit des régimes présentiels et relationnels particuliers compliquant le mouvement qui tend l'être vers autrui.

Sur la question des rapports amoureux et de leurs technologies, la littérature scientifique est rare. Les travaux d'Eva Illouz (2012) demeurent une référence centrale. Son étude sociohistorique tente de cerner l'organisation sociale de l'amour. Elle met en rapport les conceptions de l'amour avec un contexte social donné. De fait, elle discute avec pertinence les formes que revêt aujourd'hui l'état amoureux dans le contexte du capitalisme avancé. Au-delà des changements culturels et des cadres interprétatifs qui structurent l'intelligibilité attribuée à la vie et à l'amour, toute une série de dispositifs concrets accompagne et configure l'expérience amoureuse: sites de rencontre, dispositifs psychologisants d'accompagnement des couples, médiatisation de l'ordinaire de la vie amoureuse dans les revues à succès de tout âge, etc.

Son travail est précieux pour comprendre que l'amour est avant toute chose le produit de rapports sociaux concrets. Loin de la mythologie qui attribue à l'amour les traits du hasard, de l'immédiateté, de l'émotion violente, de son caractère insondable, de la naïve rencontre mystérieuse des cœurs, l'amour est régi par des normes assez strictes émanant des structures institutionnelles dans lesquelles nous vivons. Illouz ne se contente pas de rappeler ce lieu commun. Elle décrit rigoureusement les conceptions qui accréditent une certaine 
idée de la relation amoureuse à l'intérieur des formes de vie capitalistes. Ce travail empirique illustre comment les dynamiques sociales et technologiques du capitalisme exercent des influences sur les formes que prennent les vies singulières. En revanche, elle ne se penche pas sur l'ordinaire de la relation amoureuse et son équipement technologique. Elle n'entreprend en ce sens aucune phénoménologie de la relation amoureuse.

La littérature scientifique est plus abondante lorsqu'il s'agit d'appréhender les transformations des rapports de sociabilité engendrées notamment par les technologies numériques. Les analyses abondent pour tenter de qualifier au plus près l'expérience de l'autre qu'induisent les technologies (Geser, 2004 ; Kasesniemi, 2003; Pallini, Baiocco, Schneider et Atkinson, 2014 ; pour ne citer qu'eux). Le téléphone mobile est d'ailleurs un objet de choix (Jaureguiberry, 2003 ; Mizuko et Okabe, 2005). Il s'agit alors de se pencher sur les potentialités techniques ouvertes par la téléphonie mobile dans l'organisation concrète des rapports sociaux (Labarthe, 2006 ; Martin, 2003). Des analyses minutieuses décrivent les usages de la téléphonie par certains groupes sociologiques, surtout par les adolescents (Metton, 2010). D'autres travaux se sont penchés sur les modifications des régimes de socialisation dans les espaces publics (Patrascu, 2008), ou encore sur les mutations du rapport à l'oral et à l'écrit qu'ils engendrent (Akrich, Meadel et Paravel, 2001 ; Rivière, 2002). Il a déjà longuement été discuté du principe selon lequel les messages instantanés relèvent d'un mode connecté d'entretien du lien social avec les proches en maintenant une présence en continu (Licoppe, 2002; Martin, 2007). En effet, il est désormais possible de maintenir un lien en toute circonstance. Chacun peut désormais concrétiser ses besoins impulsifs de mettre en mots ses émotions ou ses sentiments à l'égard de l'autre (Rivière, 2002).

$\mathrm{Au}$ regard de la littérature scientifique, il est peut-être nécessaire d'envisager phénoménologiquement comment les technologies sont susceptibles de contribuer à mettre en cause l'expérience amoureuse tant dans la façon dont les partenaires se rendent sensibles l'un à l'autre, que dans leurs manières de réfléchir à leur liaison. II s'agit notamment d'examiner comment les amoureux font face au silence ou à la distance de leur moitié, alors même qu'ils disposent techniquement des moyens d'apparaître l'un à l'autre. Au fond, cette hypothèse invite à caractériser les ébauches d'une nouvelle forme de vie amoureuse et d'un déplacement des attentes normatives que chacun place en elle. Ce travail ne s'efforcera toutefois pas de saisir ce déplacement des conceptions de l'amour par une étude des mutations de l'éthos amoureux contemporain; il 
s'agira plutôt de proposer une phénoménologie des technologies de médiation de la présence et de l'absence, en particulier une phénoménologie de la messagerie instantanée sur le téléphone portable.

\section{Méthodologie}

On comprend bien les difficultés méthodologiques d'une telle étude. Un travail sur les rapports amoureux approche les passions secrètes et les désirs refoulés. Les messages instantanés renferment un discours de l'intime. Or, par définition, l'intime est une relation soustraite aux regards extérieurs, ce qui pose d'emblée le problème méthodologique de sa saisie (Schmol, 2005). Cette étude, encore exploratoire, s'appuie sur un corpus de messages instantanés échangés entre neuf couples différents. Parce qu'ils font partie de nos environnements existentiels, ces couples ont accepté de nous céder leurs échanges intimes sous la forme d'archive retraçant plusieurs mois de conversations. Une analyse simple de contenu a permis de dégager un certain nombre de régularités discursives et de sujets thématisés en commun dans les couples. Nous avons par la suite couplé ce matériel empirique à une série d'entretiens semi-directifs avec l'ensemble de ces personnes afin de cerner plus précisément les significations qu'ils donnent à leur activité de communication.

Les couples étudiés partagent quelques caractéristiques communes:

- un usage quotidien de la conversation à distance ;

- les relations sont inférieures à deux ans ;

- leur âge se situe entre 25 et 35 ans ;

- les couples proviennent de milieux sociaux plutôt intellectuels (enseignants, chercheurs, journalistes, étudiants, etc.) ;

- ces couples sont hétérosexuels.

À cela, il faut ajouter que ces couples ont été informés de la démarche d'étude, de la problématique travaillée et des hypothèses de recherche. Ils ont volontairement souhaité participer à la recherche en tant qu'ils reconnaissaient éprouver des problèmes dans leurs relations en rapport direct avec leur usage du téléphone portable. Ces couples avaient donc en commun d'être en proie à des difficultés relationnelles avec 
leurs partenaires, difficultés qui se cristallisaient dans de nombreuses situations conflictuelles exacerbées par leur pratique de la communication à distance.

Cet échantillon ne prétend à aucune représentativité. II porte sur des cas limites. Cette étude est exploratoire et exige d'être confrontée à une récolte plus systématique des données. À ce stade de la réflexion, il s'agit moins de démontrer la validité d'une interprétation que de figurer ou d'évoquer les possibilités inscrites dans le phénomène analysé. L'intention est de figurer une forme plausible du "devenir amoureux » et d'étayer ainsi l'imagination théorique pour saisir ce que ces médiations techniques font au rapport amoureux. En bref, le but est moins de démontrer que d'interroger le devenir du rapport amoureux à l'aune de son équipement technologique. C'est aussi la raison pour laquelle il a été décidé d'adopter une lecture de type phénoménologique. En effet, travailler à partir d'archive ne permet pas de restituer la manière dont les données se sont communiquées dans le courant de l'expérience. En d'autres termes, le corpus de messages instantanés ne retrace pas en soi le chemin du phénomène. C'est pourquoi une approche descriptive de type phénoménologique, couplée à une série d'entretiens, permet d'approcher ce matériel non comme un simple ensemble de données, mais aussi et surtout comme un ensemble de gestes (geste d'écriture, de lecture) précédant et succédant la manifestation des données. L'approche phénoménologique a donc ceci de particulier qu'elle permet de cerner les modalités par lesquelles les données s'offrent à la perception des individus, offre s'effectuant toujours dans le cadre d'un mouvement vivant que la tradition phénoménologique aide à comprendre.

\section{Les tyrannies de l'ubiquité}

Le premier trait de cette phénoménologie, le plus essentiel peut-être, a trait aux régimes de coprésence particuliers auxquels dispose le téléphone mobile. Le propre du téléphone portable est en effet de nous situer en permanence dans le " champ de portée " de l'autre - en même temps qu'il situe l'autre dans notre champ de portée propre. Le rapport à l'autre ne saurait être différé, de sorte que chacun peut s'épancher auprès de son partenaire en tout temps et en tout lieu et avoir ainsi pour visée illusoire d'expérimenter la complétude de l'autre. Comme l'indique Grégoire Chamayou (2014), « la coprésence suppose, au-delà de la simple existence simultanée de deux entités, l'inclusion de l'une dans le champ de portée de l'autre » (p. 335). Transporter avec soi un téléphone allumé, 
c'est accepter d'être " inclus dans au moins un champ de portée de l'autre ॥ (ibid., p. 335), et s'exposer aux sollicitations de tous, à tout instant et en tout lieu. C'est donc renoncer au secours de la distance en même temps qu'à ses peines. "Le principal effet des télétechnologies est [en effet] de dissocier la co-présence de sa condition de localisation » (ibid., p. 335). La relation téléphonique soustrait ainsi le besoin de la coprésence immédiate des corps à la nécessité "d'avoir du réseau ». En étant dans une zone de couverture du signal, le sujet est " offert à la présence " d'autrui, et ce, même s'il ne formule aucune intention de communication.

Chamayou (2014) introduit toutefois dans cette discussion une nuance décisive : " si la co-présence se définit comme une "présence à" elle n'est pas nécessairement une "présence pour" " (p. 336). Cette remarque est capitale. Elle rappelle que la mise à disposition dans le champ de portée d'autrui n'implique pas un partage mutuel des attentions. Qu'autrui puisse me contacter ne signifie pas qu'il le fera ni même qu'il lui viendra l'envie de le faire. De la même façon, que je puisse contacter autrui ne signifie pas que celui-ci me prêtera attention et souhaitera me répondre. Les régimes de coprésence instruits par le téléphone portable se voient ainsi situés, par la disponibilité permanente et implicite qu'ils imposent, dans un délicat jeu de tension entre la préseEce, la disponibilité d'autrui placé dans mon champ de portée, et l'abseEce, la possibilité que malgré sa "présence à " moi, autrui se refuse à être " présent pour " moi, sans que je ne sache ni ne comprenne comment ni pourquoi. C'est à peu près en ces termes qu'Édouard, en couple depuis deux ans, exprime l'anxiété suscitée par cette tension :

« II [le téléphone portable] faut pas que je l'oublie, c'est très important que je ne l'oublie pas, c'est devenu mon accessoire, peut être qu'avant on aurait pu m'offrir un porte-bonheur pour savoir que je l'ai toujours sur moi et que je pense à elle, maintenant le fait de ne plus avoir mon portable veut dire que je ne pense pas à elle. C'est-à-dire que je ne ressens pas le besoin à tout moment de pouvoir lui envoyer un message, c'est problématique, faudrait vraiment que je le mette en porte-clef, si je l'ai pas c'est un signe pour elle que y'a soucis, que je pense pas à elle, que je fais autre chose, que je la trompe, c'est la porte ouverte à toutes les angoisses. " (Extrait d'entretien avec Édouard)

Le téléphone s'apparente ici à un totem, à une sorte de talisman indispensable à la vie courante. L'essentiel n'est pas 
seulement de ne pas oublier son téléphone chez soi ; depuis longtemps, il n'y a plus d'excuses pour l'étourdi. L'important est aussi de l'avoir rechargé, d'avoir son chargeur sur soi, voire de s'enquérir d'un chargeur portatif de secours afin de pouvoir, à tout moment de la journée, interpeller son partenaire ou se rendre à sa présence selon les caprices de la situation. Le caractère anxiogène de cette tension s'exprime dans les stratégies de camouflages déployées par certains couples pour éviter tout engagement futur dans une "présence pour ». Une astuce exemplaire de cette situation est le recours au « mode avion », qui, le plus souvent, s'enclenche les pieds sur terre. Aussi involontaires soient-ils, il semble qu'en contexte amoureux, ces "épisodes d'injoignabilité » soient particulièrement mal supportés. En témoignent ces quelques échanges (Fig. 1), où le silence momentané d'un partenaire allume une cascade de harcèlements.

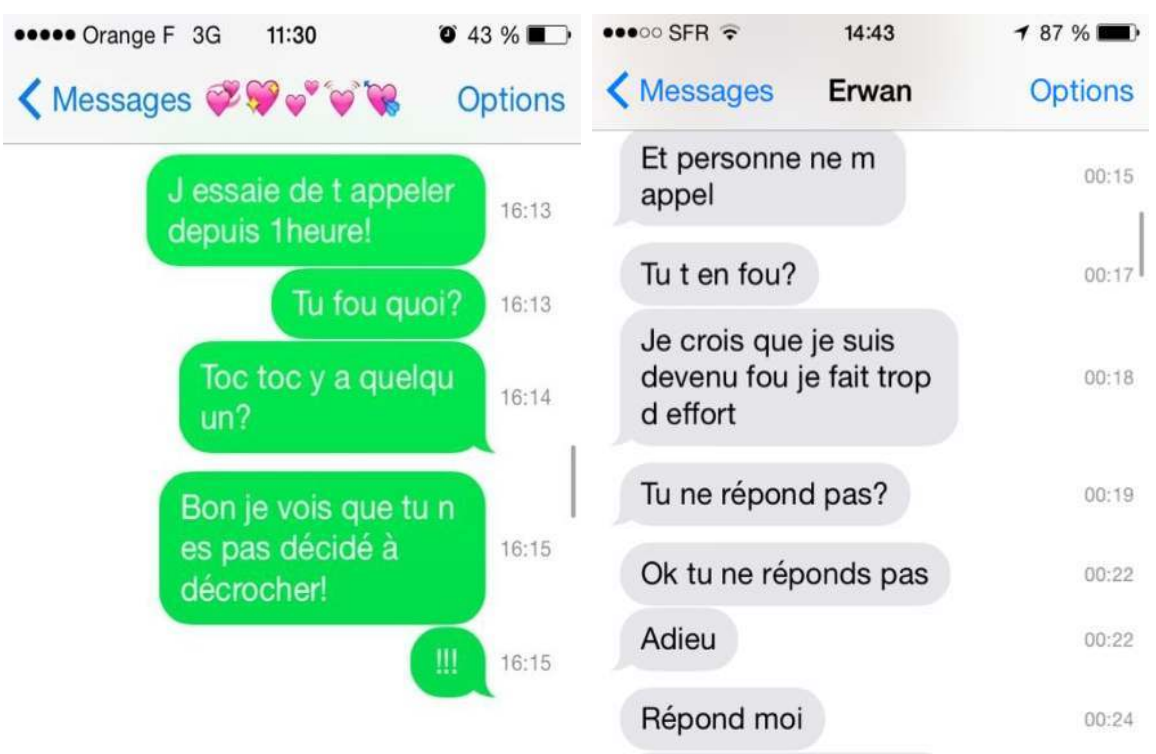

Figure 1. L'intolérance à l'absence 


\subsection{L'amour entre présence et absence}

La messagerie instantanée semble ainsi inscrire ses régimes de modalisation relationnelle au creux d'une perception particulière : la perception de l'autre comme retrait, ou plutôt la perception de l'autre comme se-retirant-de-mon-champ-deperception. Ce déchirement de la présence n'a évidemment pas attendu l'irruption du téléphone portable pour tourmenter la vie des hommes. II n'a d'ailleurs cessé, de Husserl (1996) à Nancy (1986) et de Heidegger (1927) à Blanchot (1955), de hanter la tradition phénoménologique. La phénoménologie, en effet, n'a jamais pensé la présence - à soi, à autrui, au monde - comme un donné susceptible d'être entièrement réalisé. Le monde, autrui et soi sont compris comme ce qui n'apparaît précisément que pour se refuser simultanément à livrer, dans cette apparition, une présence pleine, un sens achevé, un horizon incontestable. À cette manière qu'a le réel de résister à sa saisie, la phénoménologie a donné le beau nom "d'extériorité »-pour inscrire la séparation de cette extériorité à mon expérience et sa façon de se présenter à moi comme transcendant cette expérience.

Dans cette tradition, l'extériorité d'autrui occupe évidemment une place particulière, car l'extériorité d'autrui ne s'inscrit pas seulement dans l'extériorité d'un monde qui serait encore le mien. Elle affirme l'extériorité d'un monde autre. Ou, plus précisément, l'origiEe extérieure d'un monde autre - contestant radicalement la souveraineté du mien et de son expérience propre. Que puis-je vraiment atteindre des perceptions d'autrui, de son expérience du temps, de ses états d'âme, de ce qui occupe son attention, du rapport de son corps à sa situation ? En de nombreux cas, il nous est offert de communiquer pour essayer de réduire cet écart : un regard, un geste, une parole, un message instantané. Or quelle distance sépare encore ce que l'autre vit ou pense de ce qu'il en dit et de ce que j'en comprends ? Quelle confiance puis-je accorder à ses mots ? Et quelle évidence se perd ainsi dans la volonté de la transmettre ? La communication inscrit ainsi, simultanément, son (im)possibilité (Derrida, 1972).

La phénoménologie n'a cessé de le répéter: l'expérience d'autrui est d'abord l'expérience d'un écart entre ce qu'il m'est donné d'en percevoir (un geste, un sourire, une parole, etc.) et ce que cette perception occulte (une "forme-de-vie », un affect, un "vouloir-dire », une intériorité, etc.). La rencontre avec autrui est toujours peu ou prou l'expérience d'une absence. Or cette absence est vouée à contrarier tout effort de la présence, car celle-ci ne se laisse pas résoudre par un redoublement de la présence sensible, que celle-ci implique la coprésence des corps, l'écho de la voix ou l'échange à distance 
de messages instantanés. Et pour cause, cette absence-ci n'est pas une simple négation de la présence, mais une manière spécifique, pour les êtres, de s'apparaître, dans un inévitable retrait vers les sombres replis de ce qui échappe au savoir même infraréflexif - des choses.

Ce retrait est toutefois vécu, dans la plupart des cas, sans affection particulière. À qui importe, après tout, de savoir ce que pense vraiment son boulanger, ce qu'éprouve véritablement son garagiste, ou ce qui occupe, dans la retraite de leur intimité, l'esprit vagabond des anonymes du métro ? La situation est toutefois différente lorsqu'elle concerne une relation amoureuse. Celle-ci est marquée par certains couples comme un mystérieux désir de fusion. L'amour, et le rapport sexuel qui le signe, paraît ainsi trouver son principe dans le fantasme d'une (impossible) unité des corps et de leurs mondes. Pourtant, cette fusion est (mal)heureusement destinée à échouer. Cet échec tient dans le fait que le désir n'est effectif qu'en direction de ce qui lui échappe :

"Cela que le désir convoite et qui le satisfait se donne donc, en sa présence même, comme l'absence de ce qui ne peut en aucun cas être présent, et c'est pourquoi la satisfaction est insatisfaction: l'excès du désir, renouvelé en chaque jouissance, répond au retrait du désiré derrière ce qui la suscite " (Barbaras, 2006, p. 137).

Le « rapport " sexuel lui-même est ainsi conditionné par la séparation des êtres et des corps qu'il met en rapport. Et pour cause, il n'y a de rapports qu'entre des termes désunis (Nancy, 2001). Mais ce trait interne au désir est également redoublé d'un obstacle externe : quelle que soit l'intimité entre les êtres et le désir qui les rapproche, la séparation et l'(im)possibilité de leur communication reste un fait insurmontable. Un fait qui, dans le rapport amoureux, peut parfois provoquer de douloureuses épreuves. L'être aimé pense-t-il à moi en cet instant où je pense à lui ? M'aime-t-il autant que je l'aime ? Son esprit est-il occupé à d'autres choses lorsque mon attention lui est entièrement consacrée ? Son désir est-il orienté avec autant d'intensité vers moi que le mien n'est orienté vers lui ? Le doute soulevé par ces questions peut alors susciter une fâcheuse tendance à vouloir vérifier, à tout prix, une unité invérifiable.

\subsection{Vérifier une unité invérifiable}

$\mathrm{Au}$ sein des couples étudiés, il n'est pas rare que les amoureux s'obstinent à s'assurer, chacun pour soi ou 
mutuellement, de l'attention de l'autre. Que le partenaire travaille, lise, se repose, s'adonne à des activités sociales, l'intensité du rapport amoureux, ou la simple manifestation de sa présence, "doit " toujours s'exprimer, et chacun est mutuellement tenu de se rappeler à l'autre. Cela se traduit par de multiples interpellations anodines, comme en témoignent ces extraits typiques de messages instantanés transmis au sein des couples étudiés: "tu me manques », " je pense à toi », « $<3$ », " j'ai envie de toi », « tu fais quoi ? », " j'ai hâte de te retrouver $»$, etc.

Ces messages sont des moyens de se manifester à son partenaire, quitte à s'imposer à lui en faisant fi de son contexte. L'angoisse peut alors survenir si le partenaire ne répond pas immédiatement. À partir de là commencent les mises en crise du rapport amoureux: "? ", "??? », « Tu fais quoi ??? », "Bébé ? ", " ça va ? ", "j'espère que ça va car ta présence me manque énormément " (extraits typiques de messages instantanés transmis au sein des couples étudiés).

En ces instants où l'énonciateur est en proie au désarroi, il est tenté d'introspecter sa relation amoureuse. II cherchera alors à s'assurer du seEtimeEt de l'autre et de la pleine réciprocité du rapport. Pour s'assurer du sentiment de l'autre, le partenaire est tenu de monter en intensité ou en force l'expression de ses sentiments. II pourrait s'agir d'une sorte de mise à Eue forcée de ses émotioEs. L'extrait suivant illustre tout à fait l'escalade de l'énonciation du sentiment et des crises qu'il pourrait susciter :

S 1. 25 avr. 20:24 - Charlotte : Bebe il y a mes amies qui m'invitent pour sortir ce soir pour prendre un verre

S 2. 25 avr. 20:24 - Charlotte : Je peux y aller

S. 3. 25 avr. 20:24 - Charlotte : Et aprzs je te rejoindrai

S. 4. 25 avr. 20:25 - Mathias : Il y a mes amis qui se moquent de moi

S. 5.25 avr. $20: 25$ - Charlotte : Looooll

S. 6.25 avr. 20:25 - Mathias: Tu voulais pas sortir avec moi mais avec tes amis oui:-(

S. 7.25 avr. 20:25 - Mathias: Tu sors avec qui?

S. 8 . 25 avr. 20:25 - Charlotte : Fab et Mathieu 
S. 9.25 avr. $20: 25$ - Mathias : Ah ok

S. 10. 25 avr. 20:25 - Mathias : Bien sur mon amour on se retrouve apres

S. 11. 25 avr. 20:25 - Charlotte : Ok bebe ca marche

S. 12. 25 avr. 20:25 - Charlotte : Je t aime

S. 13. 25 avr. 20:26 - Mathias : Mais c est pas gentil quand meme: $\diamond$

S. 14. 25 avr. 20:26 - Charlotte : ?

S. 15. 25 avr. 20:26 - Mathias : Vous allez où ?

S. 16. 25 avr. 20:26 - Charlotte : On sait pas encorz

S. 17. 25 avr. 20:26 - Mathias : Ben avec moi tu as la flegme mais avec tes amies tu sors

S. 18. 25 avr. 20:27 - Charlotte : Ben en faite je vais te rejoindre c'est sur mais d'abord je vais prendre un verre avec eux

S. 19. 25 avr. 20:27 - Mathias : D'accord mon amour

S. 20.25 avr. 20:27 - Charlotte : Parceque la dernière fois j'ai refusé d'aller avec eux

S. 21. 25 avr. 20:27 - Mathias : Viens me rejoindre plein d'amour stp j en ai besoin

S. 22. 25 avr. 20:27 - Charlotte : Ok ca marche bebe

S. 23. 25 avr. $20: 28$ - Charlotte : Lol

S. 24. 25 avr. 20:28 - Mathias : Pourquoi lol ?

S. 25. 25 avr. 20:28 - Charlotte : Parceque j'ai dit ca marche

S. 26.25 avr. 20:28 - Mathias : Lol

S. 27. 25 avr. 20:29 - Charlotte : J aurait dit : oui bebe bien sur mon bebe d'amour je vais te rejoindre avec plein d'amoulle

S. 28. 25 avr. 20:29 - Charlotte : Lol 
Épuiser sa présence : rapports amoureux et téléphonie mobile

S. 29. 25 avr. 20:29 - Charlotte : Hihi

S. 30.25 avr. 20:29 - Mathias : Lol

S. 31.25 avr. 20:37 - Mathias : Je t'aime bébé

S. 32.25 avr. 20:37 - Mathias : Très fort même

S. 33. 25 avr. 20:38 - Charlotte : Moi aussi je t aime bebe

S. 34. 25 avr. 20:38 - Charlotte : Plus que toi meme

S. 35.25 avr. 20:38 - Charlotte : Plus que toi, meme

S. 36.25 avr. 20:39 - Mathias : ?

S. 37. 25 avr. 20:39 - Charlotte: Je t aime plus que tu me meme

S. 38.25 avr. 20:39 - Charlotte : Plus que tu m'aime

S. 39.25 avr. 20:40 - Mathias : Non absolument pas

S. 40.25 avr. $20: 40$ - Charlotte : Ben si

S. 41. 25 avr. 20:40 - Charlotte : Ca se voit pas ?

S. 42.25 avr. 20:45 - Mathias : Ça se voit que je $\mathrm{t}$ aime bien davantage que toi

S. 43. 25 avr. 20:45 - Mathias : C est même une évidence

S. 44. 25 avr. 20:50 - Mathias : Tu es sortie déjà ?

S. 45. 25 avr. 20:51 - Charlotte : Non pas encore bebe

S. 46. 25 avr. 20:51 - Charlotte : Je me suis maquillée hehe

S. 47. 25 avr. 20:51 - Charlotte : Lol nooonnn c'est moi qui t'aime !!

S. 48. 25 avr. 20:52 - Mathias : Donc tu constatés que je t aime davantage?

S. 49. 25 avr. 20:54 - Charlotte : Nonn

tic\&société - 10(2-3), 2016-2017 
S. 50.25 avr. 20:54 - Charlotte : C'est moi

S. 51.25 avr. $20: 54$ - Charlotte : :p

S. 52. 25 avr. 20:55 - Mathias : Mais c est faux bébé tu le sais

S. 53. 25 avr. 20:56 - Charlotte : Non c'est pas faux

S. 54. 25 avr. 20:56 - Charlotte : Tu le sais que je t aime 10000000000000 fois plus que toi

S. 55. 25 avr. 20:56 - Mathias : Tu es sérieuse ?

S. 56. 25 avr. 20:57 - Charlotte : Ben oui

S. 57.25 avr. 20:57 - Mathias : Dis moi pour de vrai bébé?

S. 58. 25 avr. 20:58 - Charlotte : Pour de vrai bebe

S. 59. 25 avr. 20:58 - Charlotte : Bebe

S. 60.25 avr. 20:58 - Charlotte : Est ce que t'as un briquet?

S. 61.25 avr. 20:58 - Charlotte : Chez toi

S. 62.25 avr. 20:58 - Mathias : J en sais rien

S. 63.25 avr. 20:58 - Charlotte : Huffff

S. 64.25 avr. 20:59 - Mathias : Pour de vrai penses tu que tu $\mathrm{m}$ aimes davantage que moi ?

S. 65. 25 avr. 20:59 - Charlotte : Oui bebe

S. 66.25 avr. 20:59 - Mathias : Sur le meuble du salon

S. 67.25 avr. 20:59 - Charlotte : Tu sais ps ?

S. 68.25 avr. 20:59 - Mathias : Je te pose la question

S. 69. 25 avr. 21:00 - Charlotte : Ben je suis sérieuse que c'est vrai

S. 70.25 avr. 21:00 - Mathias : Pour de vrai tu penses que tu $\mathrm{m}$ aimes plus que je t'aime?

S. 71.25 avr. 21:03 - Mathias : ? 
S. 72.25 avr. 21:04 - Charlotte : Oui

S. 73.25 avr. 21:04 - Charlotte : Hmm non

S. 74. 25 avr. 21:04 - Charlotte : On s'aime

S. 75. 25 avr. 21:04 - Charlotte : :)

S. 76. 25 avr. 21:04 - Mathias : D'accord

S. 77.25 avr. 21:04 - Mathias : :-)

\subsection{Exigence à la parole, à dire, à se dire}

Dans cet échange, anodin et fréquent dans ce couple ${ }^{1}$, l'instabilité ou l'insécurité du partenaire se manifeste selon au moins trois modalités :

- Par un contrôle de l'activité de son partenaire : « avec qui tu sors ? ", "vous allez où ? ", " que fais-tu ? ", etc. (Séquences 7, 15, 50-58.)

- Par une demande de rassurance quant à l'amour du partenaire: "tu m'aimes ? », "Est-ce que je te manque ? » (Séquences 13, 17, 21, 64, 70.)

- Par l'expression de l'intensité du sentiment amoureux qui, en tant que tel, expliquerait et atténuerait les effets de contrôle réalisés au cours de l'échange : " je t'aime bien plus que tu ne m'aimes [...] viens me rejoindre avec plein d'amour stp, j'en ai besoin ». (Séquences 31-54, 74.)

Cet extrait exprime assez explicitement une volonté d'asservissement et de contrôle mutuel : celle de savoir où le partenaire se trouve, s'il est fidèle, s'il existe une pleine réciprocité entre eux, si la détermination de l'amour est telle que le partenaire ne connaît aucun doute. Dans un mouvement presque instantané, ce contrôle de l'autre est compensé par l'expression de sentiments d'euphorie, d'idéalité du rapport amoureux et de l'impression de fusion, comme s'il s'agissait de compenser la négativité du commencement de la conversation.

Ainsi, la fluidité et l'insécurité du sentiment amoureux se traduisent concrètement par une polarisation autour de sentiments extrêmes comme la mise en doute (" est-ce que tu m'aimes ? »), la recherche de réassurance ("Dis moi que tu m'aimes ! [...] Tu penses à moi un peu ? »), une demande de confirmation de l'intensité du sentiment de l'autre (« je t'aime plus que tu m'aimes, ça c'est sur »).

\footnotetext{
${ }^{1}$ Sur une période de douze mois, ce genre de conversations est revenu à plus de dix reprises.
}

tic\&société - 10(2-3), 2016-2017 
Cette mise en relation exprime de façon tout à fait extravagante cette demande de validation et de sécurité. De manière permanente, il s'agit de faire connaître à l'autre qu'il est le destinataire de l'amour, du soin et de la dévotion dans une communication directe. Au sein de ce couple, ce type d'échange est assez régulier. Pour les deux partenaires, les mots ne manquent jamais pour exprimer l'intensité du sentiment amoureux. Leur rapport est saturé de paroles, de sentiments, de fierté outragée de ce qu'ils sont devenus comme couple ou encore de vérification mutuelle d'une totale réciprocité entre eux. Tout est censé être communiqué et extériorisé. Les partenaires n'ânonnent jamais. Ils s'extériorisent avec les déterminations des " je t'aime ", " tu es ma vie », " tu es le centre de toute mon existence », " je ne peux pas vivre sans toi $»$, etc. ${ }^{2}$ Les discours capturent continuellement les sentiments participant de fait à une sorte de présence vertigineuse de l'être là. Pour ce couple, et cet exemple n'a rien d'exceptionnel, le téléphone fait émerger le désir d'un échange continu et permanent de purs mots, d'une manifestation totale des indices de sa présence à l'autre. Quelles que soient les situations dans lesquelles sont engagés les partenaires, leur attention est constamment focalisée sur l'autre. II y a comme une réponse technique aux exigences pulsionnelles de l'individu qui ne peut envisager une déprise de l'autre ou être dépris par l'autre.

En outre, dans cet extrait, Charlotte demande à Mathias si elle peut sortir avec ses amis (séquences 1 et 2). Mathias se vexe, car il a le sentiment que Charlotte préfère être avec ses amis puisqu'elle avait auparavant refusé de le rejoindre (séquences 6 et 17). Mais Mathias éprouve une honte de son sentiment et, dans le mouvement, adresse une réponse des plus conventionnelles: "bien sûr mon amour, on se retrouve après » (séquence 10). Aussitôt, dans la minute qui suit et lors de son prochain tour de parole, il précipite la conversation dans une logique de mise en crise et de reproche ou de doute quant aux intentions de Charlotte: "Mais, c'est pas gentil quand même " (séquence 13). Cet échange est assez révélateur d'une oscillation entre la volonté d'adresser au partenaire une réponse ouverte, convenue et "normale », c'est-à-dire en acceptant sa séparation à soi et, dans le même temps, presque instantanément, l'incapacité concrète à assumer cette séparation, conduisant alors l'amoureux à vérifier "malgré tout " une unité que cette séparation est venue contrarier.

Enfin, cet extrait témoigne également d'une disjonction des temporalités entre les partenaires. En effet, il y a un

\footnotetext{
${ }^{2}$ Messages typiques dont l'occurrence est quasi quotidienne.
} 
entrelacement entre les conversations banales (« est-ce que tu as un briquet ? " [séquence 60], " oui sur le meuble du salon » [séquence 66]) et les conversations passionnelles ("Pour de vrai, penses que tu m'aimes davantage que moi ? » [séquence 64]). Cette disjonction des temporalités crée une coupure entre les régimes d'énonciation et est susceptible d'objectiver encore davantage la séparation entre les deux êtres. En effet, à cette demande de briquet, l'interlocuteur aurait pu s'offenser de l'intérêt pour une chose si banale dans un moment sérieux de la conversation. II aurait alors pu dire une phrase du type: "Je te dis que je t'aime alors que toi tu me parles d'un briquet ».

\subsection{Répétition et intolérance à l'absence}

Au-delà de ce cas singulier, on remarque que les messages au sein des couples se cumulent de manière vertigineuse. Les couples sont enclins à se transmettre plus d'une centaine de messages par jour pour témoigner de leurs sentiments et de leur sensation de manque de l'autre. Ces conversations ont quelque chose de répétitif puisque le répertoire linguistique exprimant le désir de l'autre n'est pas inépuisable. II existe une impressionnante régularité du lexique utilisé exprimant généralement le désir de la présence de l'autre: " tu me manques », "je t'aime passionnément », «tu es ma vie » (messages typiques repérés dans les conversations au sein des couples étudiés).

Or les amoureux sont précisément tenus de parler de leurs états d'âme et du ressenti de l'absence de l'autre plusieurs fois par jour. De fait, les mots se répètent jour après jour dans une étonnante régularité et témoignent probablement d'une tendance rationaliste dans l'expression de ces sentiments. Cette régularité du témoignage de ses sentiments est vécue par les amoureux comme préférable au silence et à la réserve. Ce langage construit le réseau sémantique de la passion illimitée et ne tolère aucunement l'absence de l'autre. De manière générale d'ailleurs, l'absence et le silence sont tenus pour être insupportables. Les messages envoyés sont alors l'instant d'une pensée ou d'une obligation à penser à l'autre : «Pourquoi tu ne m'as pas écrit aujourd'hui ? Je te trouve froid, tu n'es pas intense avec moi, tu ne penses pas à moi », " tu dois être occupé ", "Tu as envie de moi ? ça fait longtemps que tu ne me parles pas de tes désirs " (messages typiques repérés dans les conversations au sein des couples étudiés).

Cette intolérance au silence montre que les partenaires occultent tous les messages ou les temps vécus accumulés comme si seule l'actualisation du présent déterminait la solidité 
du rapport. Le rapport amoureux est tenu de s'actualiser dans tous les instants. II existe comme une perte d'évidence de la consistance du rapport amoureux. Cette actualisation permanente du sentiment amoureux est aussi un moyen de le fragiliser et de l'indéterminer. Le rapport amoureux est tenu d'être confirmé perpétuellement. Or, puisque rien ne garantit la stabilité de cette confirmation, il n'est qu'une perpétuelle attente contractée et déçue. Le rapport s'enferme alors dans sa seule logique technique qui enjoint à l'expression de soi, de l'intensité de ses sentiments amoureux et de ses états d'âme. Le rapport s'écrase dans l'instantané du clavardage. Comme les extraits de conversation le montrent, l'exigence de la présence à l'autre prend parfois des tournures frustrantes, des tensions, voire des obsessions. Le téléphone propose ici une forme d'imaginaire de la relation sentimentale bien particulière: celle de la communication illimitée, du recours systématique au langage, de l'extériorisation de soi et de ses envies, des attentes continues de confirmation mutuelle et de l'exigence d'une réflexivité permanente du couple.

Pour exemple, dans les neuf couples étudiés, il ne peut se passer plus de deux heures sans qu'un rapport soit noué entre les partenaires. Au-delà de ces deux heures, l'absence de relation s'explique par un problème technique (problème de batterie, téléphone oublié, pas de réseau) ou par une crise ponctuelle du couple. Ainsi, la technologisation du rapport amoureux implique que l'amour soit constamment mis à l'épreuve dans un processus continu et incessant de confirmation mutuelle. En quelque sorte, la technologie crée une insécurité ontologique. Le caractère incertain de la relation est désormais susceptible de s'éprouver à chaque instant, si bien que la demande de confirmation est communicable à l'envie, quelles que soient les circonstances. Concrètement, il s'y exprime une demande ininterrompue de signes et de témoignages de l'amour, comme si la relation ne pouvait et ne devait jamais être acquise. Dans cette perpétuelle et continue demande de confirmation jaillit toute une série d'angoisses ponctuelles dans les moments où la coprésence échoue.

Ainsi, le rapport amoureux devient une expérience hyperréflexive, dans la mesure où les conditions originelles qui unissent les êtres sont toujours redites et reconstituées. Le rapport est contrôlé par l'intellect et, en certaines occasions, il se manifeste de façon méthodique par une question du type : " pourquoi tu m'aimes? " ${ }^{3}$.

\footnotetext{
${ }^{3}$ Cette question est apparue au sein de trois couples différents. Elle appelle à clarifier et à objectiver les raisons de l'amour, quand bien même les réponses font
} 


\subsection{Textualité et présence}

Cependant, si les amoureux sont pareillement enclins à se solliciter sans cesse l'un l'autre, ce n'est pas seulement parce que le téléphone les pousse à vérifier mutuellement que l'autre est "présent pour eux ». C'est aussi parce que cette " présence pour » eux est, même lorsqu'elle se manifeste, incomplète et superficielle: rabattue sur l'espace lisse de la présence textuelle. Or la présence textuelle manifeste, dans sa matière même, quelque chose de l'ordre de l'insuffisance. Rien en elle n'offre l'épaisseur d'une présence physique ni même la chaleur rassurante d'une voix familière. L'écrit, en effet, présente la particularité de donner à lire une parole impersonnelle (sans aucun corps pour l'assumer) et acontextualisée. Comme le souligne Renaud Barbaras (1983), l'écrit est comme une " communication virtuelle, parole de $X$ à $X$, qui n'est portée par aucun sujet vivant et appartient par principe à tous, évoque une parole totale, métamorphose définitivement en être idéal le sens des paroles, et transforme d'ailleurs la sociabilité humaine » (p. 342). Que reste-t-il vraiment d'une personne dans ses messages instantanés? Que reste-t-il de l'intensité réelle de ses intentions ? Que restet-il de la vérité de ses affects? Rien, sinon une suite de mots autosuffisants et anonymes dont le sens ne dit rien de l'intériorité réelle de celui qui les a écrits.

L'écrit tend ainsi à effacer son auteur pour ne plus le laisser exister qu'à l'état d'énigme. Emmanuel Levinas (1994) parlait à ce titre d'un " entre temps », c'est-à-dire d'un " instant impersonnel et anonyme » (p. 138). Si sa réflexion était en premier lieu d'ordre esthétique, il n'empêche que la présence phénoménologique propre à l'œuvre d'art n'est pas sans accointances avec celle de l'écrit, car l'écriture est aussi une « présence qui ne se recouvre pas elle-même et qui se déborde de tous côtés, qui ne tient pas en main les fils de la marionnette qu'elle est » (ibid., p. 782).

Cette inaccessibilité d'autrui à travers l'écrit introduit une distance insurmontable dans les relations interpersonnelles. Comme si les mots, loin d'apporter à l'autre la certitude d'une présence et d'une attention, manifestaient à l'inverse, par l'indifférente extériorité de ce qu'ils disent à celui qui parle, l'inéluctable distance de l'être aimé. Dès lors, le service de messagerie du téléphone portable revêt les apparences d'un médiateur abstrait, car il dispose une absence inévitable de

généralement état d'un sentiment indiscernable, indéchiffrable, que les mots ne pourront sans doute pas épuiser.

tic\&société - 10(2-3), 2016-2017 
l'autre dans l'échange: le dispositif organise une communication entre individus mutuellement inscrutables.

En d'autres termes, lire un message instantané ne permet pas vraiment de savoir dans quelle disposition la personne l'a écrit : qu'était-elle en train de faire à ce moment-là ? Est-ce qu'elle pensait vraiment à son partenaire ? Quel était effectivement son état d'esprit ? Occupiez-vous vraiment son monde?

Le discours de Thibault évoque bien les situations d'incompréhension que les messages instantanés peuvent susciter :

" Au fur et à mesure des messages y'a un tout petit truc qui est pas compris qui amène l'autre à penser, à prendre une voie de pensée et du coup ça se répercute tout à la fin de la conversation, y'a un moment où on s'est tellement mal compris sur chacun des messages que moi je pense par exemple qu'elle peut être agressive alors qu'elle l'est pas, elle pense que je m'en fous alors que c'est pas vrai, ça dépend ça peut être un signe de ponctuation, ça peut être un mot qui a été employé et cetera. "

L'écrit déploie un plan de coprésentialité pauvre où l'intrication des mondes se laisse lire avec beaucoup moins d'immédiateté que dans la coprésence physique (dans l'épreuve d'un regard par exemple), ou même dans le partage de la voix. L'écrit produit ainsi l'expérience d'un monde qui n'a plus à être visé par personne. En témoigne ce passage où l'expression écrite d'une volonté de coprésence physique est immédiatement mise en doute :

$$
\begin{aligned}
& \text { Melissa : Ok bon je reste chez moi alors } \\
& \text { Fabien : :-( je veux te voir moi! } \\
& \begin{array}{l}
\text { Melissa : Non c'est pas vrai! Tu penses a peine a } \\
\text { moi }
\end{array}
\end{aligned}
$$

Cet échange montre que, tandis que le partenaire se tourne vers l'être aimé, l'écriture - le mode de présence du message instantané - vient réintroduire entre le destinataire et l'émetteur un monde qui n'appartient à personne. Dès lors, on peut discerner une tension dans ces dispositifs entre la propension qu'ils offrent de rendre accessible à tout instant la présence d'autrui et, en même temps, la pauvreté des formes de présence qu'ils rendent disponibles. Cependant, cette pauvreté 
de l'écrit est en partie comblée par le dispositif, et ce, en raison des temporalités qu'il organise.

\subsection{Tendance technique à l'accélération}

Aujourd'hui, il est difficile d'apprécier la vitesse avec laquelle se réalisent les communications. Difficile, en effet, de chronométrer avec précision le temps des médiations numériques qui s'exécutent au quotidien sur les messageries instantanées. L'intervalle entre l'envoi et la réception d'un message se dispense le plus souvent de l'affichage d'un quelconque "chargement ". L'édifice informatique, responsable de nos proximités virtuelles, ne se manifeste que sous l'émission d'un léger " plop », sonorité discrète qui indique pour mieux cacher. Impossible pour le quidam d'ausculter, d'observer ou de constater, à la suite d'un mouvement du pouce, l'orchestre mathématique jouant la note. "Instantanéité " est le nom de scène que notre modernité technologique a donné à cette invisible partition. Cette temporalité désigne en réalité une série de fragmentations/recombinaisons de données insaisissables dans leur durée. Un calcul tellement rapide qu'il devient invisible, car la vitesse vertigineuse du transfert d'informations a pour corollaire un oubli de sa présence. Le "plop » de nos messageries marque la clôture de cet imprésentable concert algorithmique : par un signal innocent, il indique à nos sens ce que la raison ne peut se représenter.

En témoigne ici la membrure invisible du « plop », où un simple « bonjour » s'enterre dans un devenir asignifiant :

$$
\begin{aligned}
& \text { <sms protocol="0" address }="+3360338 \# \# " \\
& \text { date }=" 1411117190584 " \quad \text { type }=" 1 " \quad \text { subject="null" } \\
& \text { body="Bonjour" toa="null" sc toa="null" } \\
& \text { service_center="+33609003\#\#\#" read="1" status="- } \\
& 1 \text { " locked }=" 0 " \text { date_sent }=" 0 " \text { readable_date }=" 19 \\
& \text { sept. } 2014 \text { 10:59:50" contact_name="Edouard" /> }
\end{aligned}
$$

Définir l'instantanéité comme une médiation invisible est une façon de dégager plus clairement le sol de nos usages quotidiens. Cependant, le fait que la computation soit phénoménologiquement imperceptible est insuffisant à lui seul pour comprendre l'accélération de nos discussions numériques. En effet, la célérité de nos médiations est combinée à la grande porosité des supports d'inscription. Jamais le téléphone n'indique à l'émetteur obsessionnel « vous envoyez trop de messages en même temps », freinant par ce mot d'ordre son ardent désir de communication. Les services de messagerie sont d'une perméabilité libre, car ils autorisent dans le cours du 
temps des transports de textes extrêmement rapprochés. Si cette non-limitation des flux d'informations confère à l'usager une agréable sensation de fluidité, elle rend aussi possibles les épisodes d'agression. L'hospitalité sans bornes du dispositif introduit la possibilité d'un excès, d'une surcharge de messages. Abondance admise par le support, puisque celui-ci accueille sans modération les messages envoyés par autrui.

En témoigne cette forme aujourd'hui bien connue "d'insistance computationnelle " ${ }^{4}$ où la fonction " copier/coller » matérialise l'obstination dans une série de clonages immodérée (Fig. 2).

\footnotetext{
${ }^{4}$ Nous la nommerons ainsi faute d'appellation contrôlée.
} 


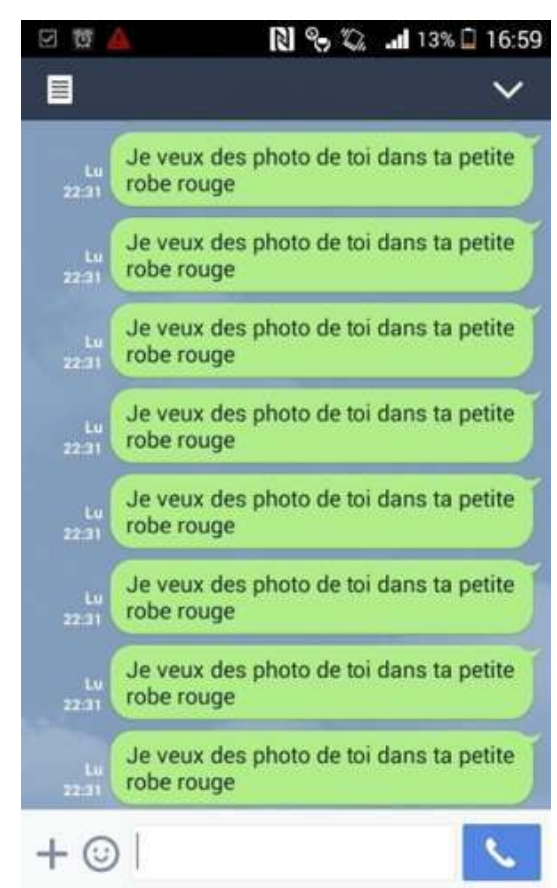

Figure 2. l'insistance computationnelle

C'est donc par la conjugaison d'une perméabilité EoE sélective eE fréqueEces d'émissioEs propre au substrat physique d'inscription et d'une médiation phénoménologiquement imperceptible que s'organise une teEdaEce techEique à l'accélératioE. Cette tendance permet un " accroissement des messages par unité de temps » (Rosa, 2005 , p. 103), en autorisant dans un délai bref l'écoulement rapide d'une multitude de notifications. Dès lors, ce qui augmente avec ces dispositifs, ce n'est pas uniquement la vitesse de transmission des messages, mais bien «la quantité des informations transmissibles par unité de temps dans un médium déterminé » (ibid., p. 103).

\subsection{Persévérance et obstination}

L'accroissement des messages par unité de temps et l'ubiquité de tous les instants font des services de messagerie des technologies numériques du «tout, tout de suite, tout le temps ॥. Cependant, la coprésence organisée par la téléphonie mobile n'est jamais pleine et entière, car rien ne garantit qu'autrui est bien présent à tout moment. Les télétechnologies façonnent ainsi l'expérience d'une présence mutuelle tressée dans l'insécurité. L'échange par messagerie altère le " présentvivant » de la relation ordinaire, en situant la discussion dans un "quasi-présent quasi-vivant », où le lien de présence n'est 
jamais acquis, mais est à entretenir en permanence. Pour maintenir la coprésence au plus près du " présent-vivant » de la relation ordinaire, le dispositif technique conduit à des régimes d'écriture que l'on pourrait qualifier de «phatique frénétique ». Dans ceux-ci, la distance est comblée par une production d'écrits qui s'effectue au détriment du contenu. L'agir prend le pas sur le propos et, finalement, l'impératif d'échanger pourrait triompher sur le sens du discours.

Dès lors, il n'est pas étonnant d'observer chez les couples modernes ces épisodes d'écriture où la production de messages est étroitement articulée à la volonté de sécuriser l'engagement affectif d'autrui : sa présence inaliénable auprès de l'être désiré. En contexte amoureux, le «phatique frénétique » exprime le plus souvent une recherche obstinée de réassurance. Transport du langage à grande distance, la téléphonie mobile pallie alors l'impossibilité du geste tendre.

En témoigne ici ce long moment d'absence où l'écrivant, enrôlé dans les angoisses de l'éloignement, exprime son besoin d'autrui dans une esthétique du morcellement :

S. 1. 23:10 - Kevin : Tu fais quoi ?

S. 2. 23:10 - Kevin : Vous sortez en ville après ?

S. 3. 23:26 - Kevin : Et voilà je suis célibataire :-(

S. 4. 23:49 - Kevin : Et bien voilà, tu m'as trompé

S. 5. 23:51 - Kevin : Quelques coupes de vin blanc et une chaleur tonitruante couplé à la rencontre. $\mathrm{d}$ un viel ami auront eu raison de moi

S. 6. 23:58 - Kevin : Lise il est une heure du matin tu bois du vin blanc avec un copain et tu ne $m$ à pas répondu depuis deux heures, vu comment tu tiens I alcool! Je crois que.....

S. 7. 24:02 - Kevin : Appel annulé

S. 8. 24:02 - Kevin : Bon.....

S. 9. 24:07 - Kevin : Étant donné que c est toi même qu une simple amitié garçon-fille $n$ était pas possible et qu il y avait toujours de la séduction dans ce type de relation tu comprend ce que je peux penser du silence actuel 
Épuiser sa présence : rapports amoureux et téléphonie mobile

S. 10. 24:08 - Kevin : "je vais voir un "vieux copain" je bois du vin blanc avec lui (ce qui est déjà beaucoup) et je ne répond plus après !

S. 11. 24:09 - Kevin : C est toi même qui $m$ à dit que les y avais pas d amitié homme femme !!!!!!!

S. 12. 24:10 - Kevin : Je comprend mieux pourquoi tu étais si enthousiaste à ton arrivé à Istanbul !!!

S. 13. 24:11 - Kevin : Tu me dira comment il embrasse comme même je veux savoir

S. 14. 24:15 - Kevin : Je regarde les messages $\mathrm{d}$ avant et bien il $\mathrm{n}$ y a jamais eu d écart de plus deux heures dans échanges

S. 15. 24:15 - Kevin : Tu vas me dire "j avais plus de batterie" mais c est pas vrai

S. 16. 24:15 - Kevin :

Liseeeeeeeeeeeeeeeeeeeeeee

S. 17. 24:16 - Kevin : Je veux savoir la vérité

S. 18. 24:18 - Kevin : Tu mettre des.doutes de côté oui je suis jaloux

S. 19. 24:19 - Kevin :Je suis un gros jaloux

S. 20. 24:19 - Kevin : Jaloux de celui avec qui tu es maintenant

S. 21. 24:19 - Kevin : Ça fait beaucoup de.choses. :-(

S. 22. 24:20 - Kevin : Je vais refaire ma vie maintenant

S. 23. 24:20 - Kevin : Demain est un nouveau jour pour moi

S. 24. 24:20 - Kevin : En plus si seulement c était une histoire de batterie tu $\mathrm{M}$ aurais prévenu :-(

S. 25. 24:21 - Kevin : Mais non la je n existe juste pas

S. 26. 24:21 - Kevin : Pas une seule nouvelle 


\section{Alexandre ROUXEL, Romain Huët et Olivier SARROUY}

S. 27. 24:22 - Kevin : Tu es avec lui maintenant, $\mathrm{d}$ ailleurs toi comme savons bien que un garçon $\mathrm{n}$ invite pas une fille juste pour de I amitié et que une fille ne rejoint pas un garçon juste pour discuter

S. 28. 24:22 - Kevin : J aurais au moins appris ça avec toi

S. 29. 24:25 - Kevin : Vous avez aimé une publication.

S. 30. 24:27 - Kevin : Pas de réponse

S. 31. 24:28 - Kevin : Pas de réponse

S. 32. 24:29 - Kevin : Pas de réponse

S. 33. 24:30 - Kevin : Tu vois line peut même indiqué "pas de réponse"

S. 34. 24:30 - Kevin : PAS DE REPONSE

S. 35. 24:30 - Kevin : Donc tu a éteint ton portable pour être tranquille

S. $36.24: 31-$ Kevin :

S. 37. 24:31 - Kevin : Je crois qu il me reste à accepter mon sort et puis $c$ est tout

S. 38. 24:32 - Kevin : Il est une heure et demi chez toi mais $\mathrm{j}$ imagine bien que ce soir tu ne dors justement pas chez toi

S. 39. 24:32 - Kevin : L.absence de réponse avant demain matin en sera la preuve la plus évidente

S. 40. 24:33 - Kevin : Merci d être honnête

S. 41. 24:36 - Kevin : Pas de réponse

S. 42. 24:41 - Kevin : Temps d'appel 0:46

S. 43. 24:41 - Kevin : Tu m'a trompé

S. 44. 24:43 - Lise :Temps d'appel 1:58

Ce passage est exemplaire du "phatique frénétique ». Le principe est de produire du contenu plutôt que d'exprimer véritablement quelque chose. L'activité « d'écriture à vide » n'a pas d'autres objectifs que de renforcer l'injonction à répondre. En marquant son engagement par une saturation de l'interface, 
l'écrivant tente désespérément d'induire un retour du partenaire. Cependant, cette création ininterrompue de contenu prend de multiples formes et le "phatique frénétique » ne se résume pas aux épisodes de solitude ni aux phases de jalousie compulsive.

\subsection{Objectiver la banalité}

En contexte amoureux, il semble en effet que la simple connaissance de notre "présence à », c'est-à-dire de notre inscription dans un champ de portée, donne lieu à des formes d' " injonctions passives ", où, coûte que coûte, il convient de marquer sa "présence pour " en écrivant quelque chose. Ces exigences de présence ont pour corollaire un appauvrissement du sens. En effet, à force d'échanger, les partenaires ne trouvent plus rien à se dire. Logé dans les poches et les sacs à main, le téléphone intime à l'écriture et à la mise en forme d'une présence pour autrui. Ce qu'il y a à dire importe peu, car il importe avant tout « d'être là, quelque part, sans toi ».

Dès lors, les énoncés phatiques se disséminent au quotidien dans de multiples insignifiances. L'actualisation permanente de la banalité est le moyen que les amoureux ont trouvé pour s'assurer, comme le disait Jackobson (1963) à propos de la fonction phatique du langage, que le circuit fonctionne bien.

Un exemple parmi tant d'autres est ce MMS (Fig. 3), où une photo stérile vient décorer un propos sans intérêt.

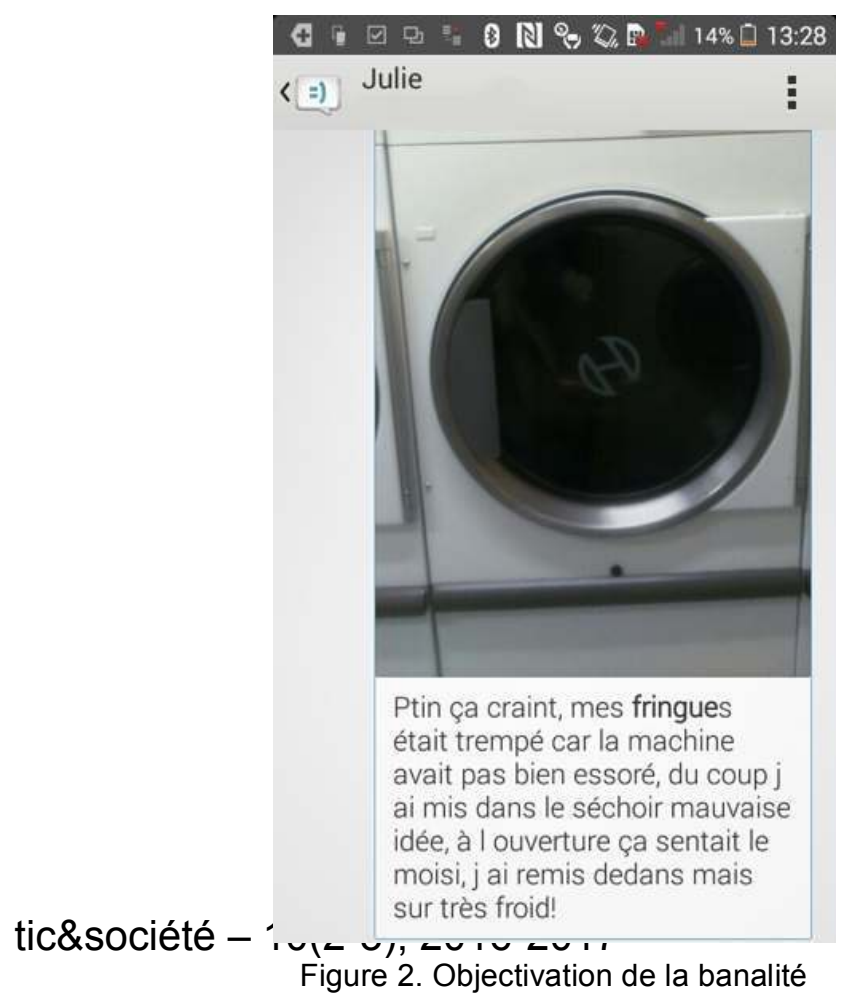


Les exemples de ce type pourraient être multipliés. L'attente d'un bus ou d'une paire de chaussettes sèches est toujours susceptible d'être objectivée dans le langage ou dans l'image, puisque dans l'éloignement, l'absence est permanente. Désormais, l'absence peut être comblée à tout moment. Vivre son couple à distance, c'est aussi s'exposer au risque de l'éprouver en comptable de l'insignifiance. C'est ce qu'explique Thibault, en couple depuis deux ans dans une relation à distance :

« J'ai l'impression que la futilité au contraire de nous rapprocher c'est quelque chose qui nous sépare, parce que justement ça détruit un peu toute forme d'intimité, ça se voudrait quelque chose qui nous rapproche, mais au final c'est quelque chose qui nous sépare, parce que ça rentre dans le commun, ça rentre dans ce que je peux raconter à mon boulanger, ou à mon buraliste, et au final ça se répète en continu et ça donne une espèce de langage routinier qui est extrêmement extrêmement chronophage, extrêmement fatiguant nerveusement. »

Évidemment, le poids des «injonctions passives » reste à démontrer et varie très certainement en fonction des couples. Cependant, à l'heure de l'accélération technique et sociale pointée par Rosa (2005), il ne serait pas étonnant de voir chez chacun ces postures du corps où, les yeux rivés sur le clavier, le dos courbé, les amoureux manifestent à l'être aimé les gestes d'existence les plus anodins.

\section{9. État de vigilance}

Sur les neuf couples analysés, les partenaires reconnaissent que leurs échanges vont régulièrement contre la raison, au sens où l'absence de l'autre est devenue insupportable. La non-réponse supposera immédiatement un "désaveu " de la relation, une occasion d'être trompé, une indifférence de l'autre. L'insistance du «tu fais quoi? tu es où? Pourquoi tu ne réponds pas ? :) » est ensuite compensée par l'expression de l'intensité du sentiment amoureux. Cette compulsion de répétition conduit les partenaires à se placer dans des situations pénibles, voire ridicules. Seule la passion est susceptible d'excuser l'excès, alors même que la relation en question n'est a priori pas nécessairement passionnelle. Seulement, la technologie pourrait précisément faire glisser 
Épuiser sa présence : rapports amoureux et téléphonie mobile

artificiellement la relation dans le champ de l'intensité passionnelle.

Au final, l'hypothèse est que le téléphone a sans doute cette propriété de fonder cette conviction d'être toujours "avec » dans cette quête sans fin. Pour reprendre les termes de Foessel (2010) dans un tout autre contexte, les partenaires sont finalement dans un état constant de « vigilance ». Cela signifie que la vigilance quant à la véracité du sentiment amoureux accompagne le quotidien. Cette forme particulière de réassurance pourrait bien fonder une relation instable, sans cesse interrogée dans ses constituants essentiels.

Dans bon nombre des échanges étudiés, les partenaires se déchirent pour manifester leur présence, mais ils constatent par suite le caractère insensé de leurs requêtes au regard des silences de l'être aimé. L'amoureux est dans une profonde " intranquilité ». D'abord peut-être parce qu'il sait bien que les régimes d'engagement sont beaucoup plus fluides et incertains qu'hier (Illouz, 2012). Mais, peut être et surtout, parce ce qu'il a les moyens empiriques de mettre à l'épreuve l'effort de l'amour. L'amoureux est tendanciellement conduit à agir sans raison. II fait fi des innombrables circonstances qui pourraient donner raison au silence de son partenaire. Or cet amoureux, finalement, n'en retiendra toujours qu'un : l'indifférence, voire la tromperie ou l'humiliation.

En ces moments, l'amoureux est seul avec son téléphone, dont il espère que la batterie ne se videra pas. II ne peut rendre raison de sa compulsion. II le fera tout de même "après coup " en expliquant que l'amour le "rend fou » et qu'il dépasse sa raison. Il calme son angoisse en se persuadant de l'intensité du sentiment et en vient d'une certaine manière à précariser encore davantage sa relation. Le présent est intensifié, étouffé par l'autre. C'est un rapport forcé, sans coupure, sans ailleurs, sans absence possible. Parfois, cette vigilance prend des formes violentes rendues possibles par la technique: se géolocaliser pour prouver où on est, prendre une photo de la situation sociale vécue, contrôler les notifications « lu, distribué, non distribué, en ligne, date et heure de la dernière connexion, etc. ". Tout un continuum de techniques produit alors cette vigilance et ce besoin compulsif de relations. Ainsi, l'expérience de l'autre comme attente et retrait, c'est-à-dire l'expérience authentique de l'autre comme énigme, est ici constamment appauvrie et déchirée par la capacité de ces dispositifs à forcer l'émergence d'une présence dénaturée :

"C'est vrai que parfois quand je regarde mes propres SMS je dois bien avouer que j'ai un peu honte de la manière dont j'ai écrit, de comment je 


\section{Alexandre ROUXEL, Romain Huët et Olivier SARROUY}

me comporte avec elle. Et puis surtout le truc qui est vraiment chiant, c'est que avec les amis, enfin quand je suis en soirée je me retrouve dans des situations où je ne suis même plus avec mes potes car je passe mon temps à écrire des messages débiles dont j'en ai rien à foutre. Ça m'emmerde mais en même temps si je le fais pas je risque de rentrer dans des conflits car j'aurais pas répondu à telle ou telle sollicitation. » (Entretien avec Kévin)

«Je sais que c'est n'importe quoi tous ces sms. Mais je ne peux pas m'empêcher de lui écrire. Et puis, on s'est habitués à faire ça, donc si je n'écris pas, ça sera forcément considéré comme un désaveu de notre relation. D'ailleurs si elle ne m'écrit pas, je sais que je pète un câble. Je deviens trop angoissé, et quoi que je fasse, je vais regarder mon portable jusqu'à qu'elle m'ait répondu. Je te jure qu'en ces moments, j'ai des palpitations au cœur. Je suis dépendant de ses mots. J'ai besoin qu'elle me rassure tout le temps. » (Entretien avec Mathias)

Ce besoin compulsif de se rendre présent à l'autre est une conduite presque permanente que les partenaires finissent par s'imposer réciproquement. Sur les neuf couples, six nous confient que la durée maximale du non-échange est de deux heures. Les partenaires sont alors plongés dans un état de mobilisation permanente pour produire une certitude précaire du rapport sentimental. Le couple tend alors à s'épuiser dans ces garanties incessantes de l'intensité et de la vérité de l'amour. À l'inquiétude comme rapport originaire à l'autre répond l'interpellation permanente mais généralement déçue de l'autre. Finalement, ce qui est censé atténuer l'inquiétude étaye encore davantage les motifs de l'inquiétude. Peut-être, et c'est là une hypothèse, que cette tendance à la compulsion de la relation s'inscrit dans ce projet typique des sociétés libérales que d'avoir une maîtrise totale de la vie, volonté de maîtrise que la technologie vient ici accentuer. La technologie ouvre la possibilité de la maîtrise en même temps qu'elle tend à défaire le rapport et à susciter des angoisses inédites face au réel. Au final, cette crainte du rapport resserra " artificiellement » les liens en créant des "solidarités forcées" pour partager l'expérience de la réassurance, c'est-à-dire en forçant l'intensité des déclamations d'amour, comme le montre bien les propos de Marc :

« II m'arrive d'envoyer un message fort, impactant, parce que comme ça je sais que les attentes des 
deux côtés seront comblées, la conversation qui avait pour objectif de créer une forme d'entente au quotidien, où on se dit c'est bon on est toujours bien tous les deux, j'envoie un message fort qui était forcément attendu les autres jours, que j'ai laissé traîner, et quand j'en ai marre je l'envoie. Je craque quoi. Je joue le jeu qui m'a été imposé c'està-dire que j'envoie moi-même un flot de messages ininterrompu et je me prends littéralement au jeu, je tombe dans le piège. " (Entretien avec Lucas)

Ces réflexions semblent montrer que ces interactions amoureuses connectées favoriseraient, sans le déterminer ni l'imposer, un état de vigilance générateur d'angoisses et de vulnérabilités quotidiennes (Foessel, 2010). Plus encore, la technologie mobile pourrait bien favoriser des formes de vie irrationnelles. La relation devient aliénée et aliénante: le partenaire se trouve en position d'individu menaçant l'équilibre psychique de l'autre. La relation s'installe rapidement dans le régime de la passion, seule explication qui pourrait rendre raison à la compulsion aux yeux des partenaires. Comme Haber (2007) l'indique dans son analyse de l'aliénation subjective, cette relation devient en partie objectivement déplorable en tant qu'elle installe la « vie interpersonnelle en générale dans l'impuissance voire dans la passivité " (p. 35). C'est le cas de cet homme qui, alors que sa partenaire est en voyage, sera rivé sur son portable des soirées entières dans l'attente d'une manifestation de l'être aimé. Au cours de ces moments, il pourrait ressentir l'état d'enlisement, d'amoindrissement (formes de tristesse liées à l'absence de l'autre, diminution de la puissance d'agir) causé par l'absence de manifestation de l'autre :

« Je te jure, je n'aime tellement pas me voir comme ça. J'ai honte. Mais je ne peux pas m'empêcher de toujours regarder si elle m'a écrit. Chaque minute, je vérifie. J'ai besoin qu'elle m'écrive. Si elle me rassure, je peux me laisser aller un peu. Mais faut qu'elle m'écrive. " (Entretien avec Lionel)

La relation s'apparente alors à une vaine excitation ou à une exaltation délétère propre à détourner de l'essentiel et qui limite l'individu de manière nuisible. En ces circonstances répétées et régulières, l'individu est plongé devant de nombreux éléments de vie affective non maîtrisés, ce qui entraîne en retour un rapport à soi non clarifiant, processus autoentretenu difficile à calmer et encore davantage à arrêter. Toujours dans le prolongement de Haber (2007), la relation est subjectivement aliénée ou aliénante en tant qu'elle s'écarte considérablement du meilleur de la vitalité de l'individu, mais aussi parce qu'elle 
est soumise dans ses manifestations principales à une pression allant dans au moins deux sens: celui de la simplification (la banalisation du " je t'aime " ou du " tu me manques ») et celui de la rigidification extrême (demande compulsive de signes de l'autre).

Pour reprendre le langage de Haber (2007), il s'ensuit une altération générale des "structures de l'intersubjectivité » amoureuse: le couple devient une forme de relation sociale dans laquelle il devient impossible d'éprouver une communication au sens d'un véritable "être-avec », avec le double moment d'identification empathique et d'une distance laissant la possibilité à l'autre d'apparaître dans son hésitation et son tiraillement. Comme le dit Thibault en conclusion d'entretien :

\begin{abstract}
"Je pense qu'on a perdu un peu le sens de l'absence, le sens de se poser hors de l'autre, et de retrouver le goût des retrouvailles, de retrouver le goût de l'absence, des vraies pensées quoi des vraies pensées, en fait on formalise ou on verbalise toutes nos pensées on les envoie par message comme si on les jetait dans le vide et on attend que quelqu'un les écoute. Donc du coup c'est très fatigant, même au bout de quelques mois c'est très fatigant. » (Entretien avec Thibault)
\end{abstract}

\title{
3. Conclusion
}

Cette étude a ouvert un certain nombre de réflexions sur quelques effets suscités par l'équipement téléphonique sur la relation. Au moyen d'une approche phénoménologique des technologies numériques, il a été montré que l'impossibilité de communication entre les partenaires s'exposait de manière radicale à même les modalités de phénoménalisation des messages instantanés. En effet, par écrit, l'autre se donne toujours dans la présentation d'une absence que seuls des jeux infinis d'interprétation et d'échange donnent à sentir. Cette inscrutabilité d'autrui, couplée à une disponibilité permanente, conduit les partenaires sur les pentes d'un contrôle et d'une sollicitation constante de l'être aimé, et ce, malgré la pauvreté des formes de présence déployées par l'écrit. Paradoxalement, la mobilisation frénétique de l'autre, permise par l'intolérance aux silences et le partage des futilités quotidiennes, semble 
participer d'une montée en intensité du sentiment amoureux. Par ailleurs, les entretiens avec les différents couples laissent apparaître chez eux un état de vigilance continu générateur d'angoisses. Ceci s'explique essentiellement par cette impossibilité de sonder l'état affectif d'autrui. Les partenaires sont alors enclins à s'engager dans des mouvements d'écriture frénétiques où chacun tente de s'assurer qu'un même désir de fusion est mutuellement partagé. Cette hyperréflexivité laisse poindre sur les corps une fatigue notable induite par une verbalisation quasi systématique des pensées. Cependant, ces états de souffrance ne peuvent occulter le plaisir qu'éprouvent également les individus à échanger ainsi. En effet, si le matériel empirique présenté ici tend à mobiliser des témoignages et des extraits chargés de négativité, il convient de souligner que ces relations s'entretiennent aussi dans le partage de voluptés, d'excitations et d'affects de joie :

«Non mais l'échange sur WhatsApp c'est aussi
super cool, elle m'envoie des photos d'elle qui me
font plaisir, enfin tu vois ce que je veux dire [Rire].
Et puis moi en contrepartie je suis content de
partager ma vie quotidienne avec elle et aussi de lui
faire des compliments sur le fait qu'elle est très
belle et tout et que bon, il est vrai qu'elle me
manque terriblement, surtout avec toutes ces
photos [Rire]. Donc dans le quotidien tu vois ça
donne aussi son lot de plaisir et de petits bonheurs
même si tout ça reste dans l'écrit et les images.
Parfois on est super in-love même s'il est vrai qu'on
se prend la tête régulièrement. Mais c'est pas tout
noir ou tout blanc quoi. " (Entretien avec Thibault)

\subsection{Le message instantané ou la machine mélancolique}

II n'y a donc pas à s'alarmer, ici, des effets du téléphone portable sur l'ordre de nos relations. Certains y trouveront la source d'une douleur, d'autres, l'occasion d'une joie. II importe plutôt de saisir que ces affects s'inscrivent au creux d'une expérience identique : le scintillement minuscule de l'autre dans le lointain de sa disparition. Les messages instantanés se donnent comme le point de jonction entre la réalité et le fantasme: réalité du texte cristallisé sur l'écran d'un côté, fantasme de l'amoureux sur les états d'âme véritables de son auteur de l'autre. Dans cette dialectique apparaissent les traits caractéristiques de l'attitude mélancolique. Ce penchant 
mélancolique n'est pas propre, d'ailleurs, aux messages instantanés. II concerne plus largement l'ensemble des régimes de présence s'appuyant sur la médiation d'une trace dont le " présent-vivant » de l'inscription a été irrémédiablement perdu. Par exemple, cette analyse sur les messages instantanés partage celle proposée par Gunthert (2010) dans la Petite histoire de la photographie de Walter Benjamin: "il semble bien qu'il faille formuler l'hypothèse d'un lien étroit de la photographie avec cette forme du deuil que Freud nomme mélancolie » (p. 6). Les messages instantanés comme la photographie partagent une caractéristique commune. L'une comme l'autre s'offre comme l'expérience d'une perte dont il est difficile de préciser toutefois ce qui, au juste, a été perdu. Qu'est-ce qui, dans la photo d'un anonyme que je n'ai jamais connu, peut bien apparaître comme une perte ou une disparition ? Et qu'est-ce qui, dans ce message instantané clôt sur lui-même que m'adresse l'être aimé, peut bien apparaître comme un manque?

Freud ne s'en cachait pas. Pour lui, dans la mélancolie, une perte est bien advenue, mais sans qu'il soit possible de savoir ce qui a été perdu. Si perte il y a, elle ne peut être qu'irréelle, car le mélancolique n'enterre que des chimères. Cependant, au sein de ce jeu de contradictions où surgit l'étrange phénomène d'un deuil sans perte, nous pouvons apercevoir toute l'ambiguïté de la notion :

«Dans la mélancolie l'objet n'est ni approprié ni perdu mais approprié et perdu simultanément. De même que le fétiche est tout à la fois le signe de quelque chose et de son absence cette contradiction lui conférant son statut fantomatique, de même l'objet visé par la mélancolie est en même temps réel et irréel, incorporé et perdu, affirmé et nié » (Agamben, 1992, p. 49).

Cette citation d'Agamben témoigne de la double polarité de la mélancolie comme épiphanie d'un irréel altérant la perception de l'actuel. En effet, la mélancolie est d'abord une affaire de perception. C'est ce qui la différencie de la nostalgie, laquelle est un sentiment qui se joue dans l'espace de la mémoire pour y mener son commerce avec les souvenirs. Dès lors, le média photographique comme les messages instantanés conservent par-devers eux ce caractère mélancolique. Contempler une photo ou lire un message instantané, c'est toujours se rapporter à un inactuel mystérieusement présent dans son absence même. Or, comme nous l'avons rappelé, la mélancolie surgit et naît de l'insuffisance du visible, car elle ne se rapporte qu'à des points de fuite. Comme le mélancolique, l'appareil photographique ou les messages instantanés échouent dans 
leur appréhension du réel, car ce qu'ils tentent de capturer reste étranger à toute saisie possible. Les dispositifs de messageries instantanées partagent cette même caractéristique avec la photographie. En effet, ces « moyens technologiques" ne connaissent que des "finalités mélancoliques ». Étrange paradoxe puisque la mélancolie semble étrangère à toute idée de finalité, et ce, en vertu de l'effort permanent qu'elle demande à l'imagination.

Cependant, chaque message instantané, chaque photo, se donne comme la présentation d'une absence que seul un acte imaginaire est en capacité de rejoindre. Si finalité il y a, alors celle-ci ne peut exister que sous le mode d'une relance incessante de l'imagination s'épuisant dans son vol tout entier tendu vers l'objet inconnu et désespérément absent. Ne subsiste dans les photos et dans les "messages instantanés » qu'un apaisant « ça a été " dont on ne sait pas d'ailleurs si cela a véritablement été, ni où ni comment. Seul un esprit rêveur est en mesure de répondre à ces questions et c'est aussi pour cette raison que la mélancolie ne peut conduire, à terme, qu'à une "hypertrophie de l'imagination ». Dès lors, les technologies de communication de type message instantané fonctionnent comme des machines mélancoliques. Machine dans la mesure où elles asservissent l'individu à l'attitude mélancolique ${ }^{5}$, c'est-à-dire à cette posture de l'homme qui faute de pouvoir toucher et rejoindre ce qui paraît, reste immobile dans l'attitude contemplative. C'est pourquoi nous pouvons formuler l'hypothèse selon laquelle c'est en raison de cette dimension machinique qu'émane au sein des couples étudiés précédemment un certain type de régime affectif, que nous nommerons ici passioE. En effet, comme le souligne Agamben (1992) «le penchant irrépressible du mélancolique pour la contemplation le pousse fatalement vers la passion amoureuse » (p. 49).

\subsection{La passion ou l'individuation sans sujet}

Que ces dispositifs fonctionnent comme des machines mélancoliques les conduit en effet à attiser, chez leurs utilisateurs, une étrange émotion: la passion. Gilles Deleuze (2014) introduit ainsi, dans le commentaire qu'il propose d'un entretien entre Michel Foucault et Werner Schroeter, une distinction entre ces deux régimes d'individuation que sont l'amour et la passion: "L'amour est un état et un rapport de

\footnotetext{
${ }^{5}$ Sur la notion d'asservissemeEt et sa distinction d'avec l'assujettissemeEt, nous renvoyons le lecteur au travail de Maurizio Lazzarato (http://eipcp.net/transversal/1106/lazzarato/fr).
}

tic\&société - 10(2-3), 2016-2017 
personnes, de sujets. Mais la passion est un événement subpersonnel qui peut durer aussi longtemps qu'une vie, un champ d'intensités qui individue sans sujet »(p.64). Que la passion procède d'une individuation sans sujet pourrait sembler tout à fait contradictoire. Toute individuation affecte bien, après tout, le rapport d'un être à lui-même et à son monde. Pourtant, la passion ne réclame-t-elle pas des êtres qu'elle affecte qu'ils se rapportent à eux-mêmes et à l'autre comme à des sujets ? La passion s'adresse à des spectres - et constituent comme spectres ceux qu'elle traverse. Bien sûr, il existe bien quelque chose comme un sujet derrière chaque message. Bien sûr, ce sujet livre même souvent, dans les courts fragments de texte qu'il adresse à l'autre, le secret de ses états d'âme les plus intimes. Mais qu'un sujet partage ainsi son intimité ne signifie pas que celle-ci sera comprise comme telle ni que la réaction de l'autre confirmera la proximité de cet énoncé à son auteur.

Les messages instantanés introduisent en effet, entre les êtres passionnés, la médiation - ou l'écart - d'une abstraction. Si nous avons précédemment nommé cet équipement mobile " machine mélancolique », c'est aussi et surtout parce qu'il fonctionne comme un système de médiation abstrait. L'effacement du corps et des multiples indices affectifs qu'il supporte introduit une irréductible indétermination dans le sens des énoncés. Comment comprendre une parole quand aucune situation, aucune tonalité, aucun regard, aucun geste ne l'accompagne plus? Aussi, les messages instantanés parviennent-ils, et s'adressent-ils toujours, à un autrui a priori dont l'interlocuteur ignore tout de la polarité, c'est-à-dire de l'orientation affectivo-émotive. Tout compliment peut ainsi muter en agression et toute attention, en incivilité.

Les phénomènes d'incompréhension et d'interprétation récurrents dans les couples étudiés traduisent bien le fait que l'agentivité textuelle des messages instantanés, ainsi rendus à une autonomie indépendante du "vouloir-dire » de leur auteur, exercent des effets sémantiques et affectifs que personne ne contrôle plus et qui peuvent conduire les individus en des lieux qu'ils n'ont parfois jamais vraiment décidés d'occuper. Dans sa relation à l'opacité des états affectifs d'autrui, chaque message instantané, c'est-à-dire chaque geste adressé à l'autre, est ainsi un pari contracté avec le hasard. L'auteur ne sait jamais ce qui arrivera aux spectres qu'il a lui-même créés, dans la mesure où son action s'adresse à une pure possibilité d'autrui comme être " probablement » polarisé de telle ou telle manière. De fait, chaque message instantané, chaque parole, chaque geste est virtuellement chargé d'une profusion de lectures possibles. Personne ne sait vraiment ce qui arrivera à ses intentions numériquement médiées, et ce, en vertu de l'invisible adresse 
du désiré. Tel un boomerang fou n'ayant aucun respect pour sa trajectoire initiale, la lecture d'un message instantané ignore toujours l'intentionnalité qui l'a portée. C'est depuis cette extériorité du sens (aux intentions des sujets "écrivants ») que s'élève alors l'agencement passionnel articulé par la messagerie instantanée. C'est en effet depuis l'impossibilité de sonder et d'appréhender les points de vue d'autrui que surgit le champ d'intensité évoqué tour à tour par Deleuze et Foucault à propos de la passion. Parler de passion dans le cas qui nous préoccupe, c'est comprendre ce qui arrive aux intentions dès lors que celles-ci sont portées par la technique. Car enfin, l'intensité résulte d'une altération, d'une transformation de l'intention. L'intensité, c'est ce que devient une intention dès lors que celle-ci, dans son déploiement même, ignore l'état affectif de ce qu'elle va pourtant rencontrer, tel un geste aveugle ne sachant ce qu'il va pourtant toucher.

S'adresser à l'autre par message instantané est un pari sur une lecture dont rien ne garantit la venue. Ce qui arrivera durant le «présent-vivant » du lecteur est absolument imprévisible, et c'est pour cette raison que l'intention laisse systématiquement sa place à un foisonnement d'intensités. L'invisibilité d'autrui dans l'adresse (le moment de l'écriture comme rapport à une opacité) et dans l'adressé (le texte transmis et ses mystérieuses lectures) fait vibrer l'intentionnalité à un point d'incertitude tel qu'elle se mue en intensité. Le sens que l'auteur souhaitait donner à son texte n'appartient plus à personne, le message instantané devient pure intensité, car, dans son mouvement autonome, il contracte en lui une infinité d'effets possibles. Les messages tendent ainsi à se constituer, dans ces échanges comme le lieu de circulation d'un discours autonome assignant les interlocuteurs à une posture de passivité sur leurs propres énoncés. Dépossédé de sa parole et destitué des privilèges du locuteur, l'auteur ne peut plus, alors, se réclamer de rien. Déchu de ses intentions, il pourra alors spéculer, solitairement, sur les qualités de sa prestation.

Ne reste alors plus à l'auteur qu'une position de spectateur face à sa propre affectivité. En révoquant les intentions originelles de leurs messages mutuels, les interlocuteurs tendent en effet à révéler les forces impersonnelles qui animent le discours de l'autre et précèdent ses intentions: amour, jalousie, colère, etc. L'auteur se voit ainsi confronté, par l'autre, aux forces obscures qui l'animent et que sa conscience s'applique usuellement à retenir dans l'obscurité. Ces énoncés qu'il croyait siens, l'autre, par sa (més)interprétation, leur assigne une autre origine et les renvoie à d'autres forces. L'auteur se voit ainsi décentré de lui-même, comme affecté par ses propres paroles, qui, dans leur séparation à lui, le renvoient 
chaque fois à autre chose que lui-même. Ces énoncés si intimes que l'auteur était parvenu à soustraire au murmure informe de son intériorité, le message instantané les convertit en un cri impersonnel interrompant la proximité du rapport à soi, confrontant le " je " à l'extériorité d'un affect subpersonnel révoquant la fiction de son individualité.

C'est pour ces raisons que l'on peut dire des machines mélancoliques qu'elles créent des agencements passionnels, car, en transformant l'intention en intensité, la téléphonie mobile confronte les sujets à des devenirs qui les fondent en lieu et place de leur absence. Les machines mélancoliques sont les outils d'une désubjectivation facilitant l'émergence de la passion, "événement subpersonnel » au sein duquel, comme nous le rappelait Foucault, "ça n'a plus de sens d'être soi même ".

\section{Références bibliographiques}

AGAMBEN G.,1992, StaEze, Paris, Payot et Rivages

AKRICH M., MEADEL C. et PARAVEL V., 2001, « Le temps du mail. Écrit instantané ou oral médiat », Sociologie et Sociétés, vol. 32, $\mathrm{n}^{\circ} 2$, pp. 153-170.

BARBARAS R., 1983, L'être du phéEomèEe : sur l'oEtologie de Merleau PoEty, Paris, Jérôme Millions.

BARBARAS R., 1999, Le désir et la distaEce : iEtroductioE à uEe phéEoméEologie de la perceptioE, Paris, Vrin.

BAUMAN Z., 2004, L'amour liquide: de la fragilité des lieEs eEtre les hommes, Paris, Fayard.

BLANCHOT M., 1983, La commuEauté iEavouable, Paris, Éditions de Minuit.

DERRIDA J., 1972, Marges de la philosophie, Paris, Éditions de Minuit.

DEleuZE G., 2003, Pourparlers, Paris, Éditions de minuit, Paris.

FOESSEL M., 2010, État de vigilaEce, critique de la baEalité sécuritaire, Paris, Le Bord de l'eau.

FOUCAULT M., 2001, Dits et écrits. Tome 2 1976-1988, Gallimard, Paris.

GESER H., 2004, "Towards a Sociological Theory of the Mobile Phone ", Sociology iE SwitzerlaEd, <http://socio.ch/mobile/t_geser1.htm>, dernière 
Épuiser sa présence : rapports amoureux et téléphonie mobile

consultation le 14 mars 2017.

GUNTHERT A., 2010, « Archéologie de la "Petite histoire de la photographie" ", Images Re-vues, hors-série, <http:// imagesrevues.revues.org/292>, dernière consultation le 15 juillet 2013.

HABER S., 2007, L'aliénation. Vie sociale et expérience de la dépossession, Paris, Presses universitaires de France.

HUSSERL E., 1996, Leçons pour une phénoménologie de la conscience intime du temps, Paris, Presses universitaires de France

ILLOUZ E., 2012, Pourquoi l'amour fait mal. L'expérience amoureuse dans la modernité, Paris, Le Seuil.

JACKOBSON R., 1963, Essais de linguistique générale, Paris, Éditions de Minuit.

JAUREGUIBERRY F., 2003, Les branchés du portable, Paris, Presses universitaires de France.

KASESNIEMI E.-L., 2003, Mobile Messages: Young People and a New Communication Culture, Tampere, Tampere University Press

LABARTHE F., 2006, Ce que tchater veut dire. Pratiques internautes, Paris, Éditions du Seuil.

LEVINAS E., 1978, De l'existence à l'existant, Paris, Vrin.

LICOPPE C., 2002, "Sociabilité et technologies de communication. Deux modalités d'entretien des liens interpersonnels dans le contexte du déploiement des dispositifs de communication mobiles, Réseaux " mobiles », vol. 20, n 112-113, pp. 171-210.

MARTIN C., 2003, «Le téléphone portable: machine à communiquer du secret ou instrument de contrôle social ? ", Communication et langages, $\mathrm{n}^{\circ} 136$, pp. 92105.

METTON C., 2010, « L'autonomie relationnelle. SMS, « chat », et messagerie instantanée », Ethnologie française, vol. 40, $n^{\circ} 1$, pp. 101-107.

ITO M., 2003, « Mobile Phones, Japanese Youth, and the RePlacement of Social Contact », dans R. LING (dir.), Front Stage/Back Stage: Mobile Communication and the Renegotiation of the Public Sphere, <http://www.itofisher.com/mito/archives/mobileyouth.pdf> , dernière consultation le 15 mars 2017.

NANCY J.-L., 2001, L'« il y a » du rapport sexuel, Paris, 
Galilée.

MIZUKO I. et OKABE D., 2005, «Réseaux intimes : contextualiser la relation des jeunes japonais à la messagerie mobile », Réseaux, $\mathrm{n}^{\circ} 133$, pp. 14-43.

PALLINI S., BAIOCCO R., SCHNEIDE, B.-H. et ATKINSON L.R., 2014, "Early Child-Parent Attachment and Peer Relations: A Meta-Analysis of Recent Research », Journal of Family Psychology, vol. 28, n¹, pp. 118-123.

PATRASCU M., 2008, "L'usage de la télévision sur le téléphone mobile et des plateformes de partage de vidéos en France. Résultats d'une enquête qualitative ", Cahier de recherche, $\mathrm{n}^{\circ} 2$, <http://www.marsouin.org//MG/pdf/Patrascu_22008.pdf>, dernière consultation le 14 mars 2017.

RIVIĖRE C.-A., 2002, "La pratique du mini-message. Une double stratégie d'extériorisation et de retrait de l'intimité dans les interactions quotidiennes ", Réseaux " mobiles », vol. 20, n 112-113, pp. 139-168.

SCHMOL P., 2005 «La rencontre amoureuse: entre permanences et mutation », dans A. Touati (dir.), Femmes/Hommes. L'invention des possibles, Antibes, Sciences de l'Homme et Sociétés, pp. 137-147. 